\title{
OPEN Swedish intrauterine growth reference ranges for estimated fetal weight
}

\author{
Linda Lindström ${ }^{1 凶}$, Mårten Ageheim ${ }^{1}$, Ove Axelsson ${ }^{1,2}$, Laith Hussain-Alkhateeb ${ }^{3}$, \\ Alkistis Skalkidou ${ }^{1}$, Anna-Karin Wikström ${ }^{1}$ \& Eva Bergman ${ }^{1}$
}

Fetal growth restriction is a strong risk factor for perinatal morbidity and mortality. Reliable standards are indispensable, both to assess fetal growth and to evaluate birthweight and early postnatal growth in infants born preterm. The aim of this study was to create updated Swedish reference ranges for estimated fetal weight (EFW) from gestational week 12-42. This prospective longitudinal multicentre study included 583 women without known conditions causing aberrant fetal growth. Each woman was assigned a randomly selected protocol of five ultrasound scans from gestational week $12+3$ to $41+6$. Hadlock's 3rd formula was used to estimate fetal weight. A two-level hierarchical regression model was employed to calculate the expected median and variance, expressed in standard deviations and percentiles, for EFW. EFW was higher for males than females. The reference ranges were compared with the presently used Swedish, and international reference ranges. Our reference ranges had higher EFW than the presently used Swedish reference ranges from gestational week 33, and higher median, 2.5th and 97.5th percentiles from gestational week 24 compared with INTERGROWTH-21st. The new reference ranges can be used both for assessment of intrauterine fetal weight and growth, and early postnatal growth in children born preterm.

An important part of antenatal care is assessment of fetal growth, as growth and birthweight are important factors that influence perinatal morbidity and mortality ${ }^{1-3}$. There are different approaches in screening for fetuses with high risk of morbidity and mortality due to aberrant growth. Serial measurements of the symphysis-fundus distance can be used as a simple and inexpensive screening tool, followed by ultrasonic estimation of fetal size if deviating $^{4-6}$. Selective or universal ultrasonography are other screening tools for aberrant fetal growth. Irrespective of indication, reliable standards are vital for the evaluation of fetal size and timely recognition of aberrant growth.

A high-quality standard for fetal size and growth describes how fetuses grow under optimal conditions. Reference ranges of intrauterine growth should be based on studies with a robust design, a reasonably large cohort and use adequate statistical modelling methods for calculation of percentiles, to aid clinical judgement ${ }^{7,8}$. Children born preterm are more often growth restricted compared with children born term ${ }^{9}$. Thereby, the birthweight of children born preterm is substantially lower than the weight in children of the same gestational age (GA) who remain in utero until term ${ }^{10-12}$. Thus, relying on information of birthweight in infants born preterm to extrapolate and develop fetal weight reference ranges should be avoided; rather longitudinal intrauterine assessment of fetal weight should be used ${ }^{10,12,13}$. Moreover, the inevitable neonatal intensive care exposes the preterm born infant to a stressful extrauterine environment that affect early postnatal growth, and consequently intrauterine growth reference ranges are often recommended for monitoring early postnatal growth in preterm born infants ${ }^{13}$.

Fetal weight can be estimated using a variety of algorithms, which combine ultrasonographic assessment of biometric measurements. The most widely used algorithms include combinations of biparietal diameter (BPD), head circumference (HC), mean abdominal diameter (MAD), abdominal circumference (AC) and femur length $(\mathrm{FL})^{14}$. Several studies have evaluated these algorithms, as there is no universally accepted formula for fetal weight estimation ${ }^{15-18}$. Even though the results diverge to some extent, most studies have found Hadlock's formula, including HC, AC and FL with or without addition of BPD, superior or non-inferior to other formulae in estimating fetal weight or predicting adverse perinatal outcome ${ }^{15-19}$.

${ }^{1}$ Department of Women's and Children's Health, Uppsala University, Uppsala, Sweden. ${ }^{2}$ Centre for Clinical Research Sörmland, Uppsala University, Eskilstuna, Sweden. ${ }^{3} \mathrm{Global}$ Health, School of Public Health and Community Medicine, Institute of Medicine, Sahlgrenska Academy, University of Gothenburg, Gothenburg, Sweden. ${ }^{\square}$ email: linda.lindstrom@kbh.uu.se 
During the last years, the World Health Organization Multicentre Growth Reference Study and the INTERGROWTH-21st have presented fetal growth standards intended for universal use in all populations ${ }^{20,21}$. Due to variations in fetal size over the globe, it has been questioned if these international standards are better than national references in detecting growth restricted fetuses with increased risk of adverse outcome ${ }^{22-27}$.

The currently used Swedish growth reference ranges for estimated fetal weight (EFW) were published in 1996 by Maršál et al., and present EFW from gestational week 25 to 43. The growth reference ranges are based on 86 Scandinavian pregnant women, of whom $24 \%$ were smokers ${ }^{10}$. Today, fetal interventions can be made during the second trimester of pregnancy, and survival is possible for children born as early as 22 gestational weeks. To meet up with these obstetrical challenges, there is a need for updated national reference ranges, which reflect expected fetal growth throughout the second trimester in a representative and healthy population.

The aim of this study was to create new national reference ranges for EFW from gestational week 12 to 42 , applying modern statistical methods to a longitudinal study of ultrasonically derived intrauterine biometric measurements in a large Swedish cohort of healthy pregnant women with expected normal fetal growth. Further, we wanted to compare the new reference ranges with other relevant growth reference ranges of EFW.

\section{Methods}

Study design and population. In this prospective longitudinal multicenter study, 684 women were recruited in early pregnancy between September 2015 and September 2018 in five sites in central Sweden; Uppsala, Falun, Katrineholm, Västerås and Örebro. In Sweden, antenatal care is standardized and free of charge. All women are offered a routine second trimester ultrasound scan. The purpose of this scan is to evaluate GA (unless the pregnancy was dated during the first trimester), number of fetuses, viability, placental location and search for structural anomalies ${ }^{28}$. Women with low risk pregnancies are not routinely scanned for aberrant fetal growth with ultrasound during the third trimester of pregnancy. At first antenatal visit, all women who received antenatal care at 18 primary care units in urban as well as rural areas representing different socioeconomic and ethnic backgrounds were invited to participate in the study. Non-smoking women were eligible if they had regular menstrual periods ( $28 \pm 4$ days), a spontaneously conceived pregnancy, and no previous pregnancy complications, such as preterm birth, gestational hypertension, pre-eclampsia or eclampsia, gestational diabetes or stillbirth. Women with chronic hypertension, systemic lupus erythematosus, kidney disease, diabetes mellitus, previous gastric bypass surgery or inflammatory bowel disease were not eligible, as these conditions are known to affect fetal growth.

Eligible women were invited to the first study visit between gestational week $12+3$ and $13+6$ according to the last menstrual period. Eligibility was evaluated and GA assessed with ultrasound. Only women with singleton pregnancies with $\mathrm{BPD} \geq 21 \mathrm{~mm}$ were recruited, and GA according to BPD should not deviate more than seven days from GA according to last menstrual period. Written informed consent was obtained from all the study subjects before the dating scan was performed.

To get an even distribution of the ultrasound scans throughout pregnancy, each study subject was randomized to one of nine study protocols, with different timing of the ultrasound scans. Each protocol included five scans allocated between gestational week 14 and 41 . The study protocols were kept in closed envelopes and were randomly assigned to each woman at inclusion. In Sweden, all pregnant women are offered the routine second trimester scan at gestational week 17-20. If the woman was not randomized to a scan in week 17-20 (five protocols), the biometric measurements were recorded in the study database only if the routine second trimester scan was performed by a study sonographer. In order to compensate for the expected decline in the number of women with ongoing pregnancies, more women were assigned to the scans in gestational week 37-41.

Women were excluded from the study if the pregnancy was complicated by gestational hypertension, preeclampsia or eclampsia, gestational diabetes, single umbilical artery, placental complication such as placenta previa and placental abruption, fetal malformations or chromosomal aberrations, stillbirth, fetal growth restriction or preterm birth (before $37+0$ gestational weeks; 259 gestational days). Fetal growth restriction was defined as EFW $<-2$ standard deviations (SD) according to the reference ranges by Maršál et al. with abnormal umbilical Doppler and/or oligohydramniosis (single deepest pocket $<2 \mathrm{~cm})^{10}$.

Out of the 684 recruited women, 650 were eligible for the study. During pregnancy, 14 women (2.2\%) were excluded due to gestational hypertension or pre-eclampsia, and $11(1.7 \%)$ due to development of gestational diabetes. Six women $(0.9 \%)$ were excluded as they developed fetoplacental complications, such as placenta previa, placental abruption, single umbilical artery and preterm fetal growth restriction. One woman had a late miscarriage, one child was stillborn and 26 children (4.0\%) were born preterm. Lastly, 8 women (1.2\%) were excluded due to fetal malformations or chromosomal aberrations. Women who were excluded from the study due to pregnancy complications more often had an increased BMI; $9.9 \%$ for BMI $25.0-29.9 \mathrm{~kg} / \mathrm{m}^{2}, 23.8 \%$ for $\mathrm{BMI} \geq 30 \mathrm{~kg} / \mathrm{m}^{2}$, versus $7.7 \%$ for $\mathrm{BMI}<25 \mathrm{~kg} / \mathrm{m}^{2}$, or were born outside the Nordic countries; $15 \%$ versus $10 \%$. The final cohort consisted of 583 women; 275 from Uppsala, 66 from Falun, 98 from Katrineholm, 50 from Västerås and 94 from Örebro.

Procedures. All ultrasound scans were performed by seven experienced sonographers. The ultrasound machines used were GE Voluson E10, GE Voluson E8 and GE Voluson E6 with abdominal transducers 2-6 MHz RM6C, 2-8 MHz C4-8-D, RAB 4-8-D and 2-9 MHz C2-9-D.

Before first inclusion, all sonographers were given detailed oral and written instructions regarding how the biometric measurements should be performed. A repeatability and reproducibility study was performed, which is described in detail in a previous publication ${ }^{29}$. BPD was used to calculate the GA, using the modified Selbing and Kjessler formula, $58.65+1.07{ }^{*} \mathrm{BPD}+0.0138^{*} \mathrm{BPD}^{2}$, as recommended by the Swedish Society of Obstetrics and Gynecology $y^{30-32}$. At each ultrasound scan, the sonographer measured five biometric measurements trifold; 
$\mathrm{BPD}, \mathrm{HC}, \mathrm{MAD}, \mathrm{AC}$ and $\mathrm{FL}$, and all three measurements were registered in the data base. All data was manually registered in a web-based study database by the sonographer.

To estimate fetal weight, we used Hadlock et al.s formula 3, which includes the biometric measurements $\mathrm{BPD}, \mathrm{HC}, \mathrm{AC}$ and $\mathrm{FL}^{19}$. BPD and $\mathrm{HC}$ were measured in axial section, at the level of the thalami, with the midline echo in a central position broken anteriorly by cavum septum pellucidum. Orbitae and cerebellum should be non-visible. The callipers for BPD were placed on the outer margin of the proximal parietal bone, and the inner margin of the distal parietal bone. HC was measured by placing the callipers on the outer borders of the frontal and occipital edges of the bone, and the ellipse facility was used to follow the contours of the skull. AC was measured in cross-section, with a circular view of the abdomen, the stomach visible, the umbilical vein in the anterior third of the abdomen and the aorta and inferior vena cava anterior to the spine. Further, the majority of a rib should be seen, but not the heart or kidneys. AC was measured using the ellipse facility to follow the outer contours of the skin. FL was measured in a longitudinal section of the femur in $45^{\circ}-90^{\circ}$ angle of insonation, with the callipers placed on the outer borders of the femoral diaphysis. All measurements followed the national recommendations for biometric assessment and the practice guidelines from The International Society of Ultrasound in Obstetrics and Gynecology $y^{30,33}$.

Data management. Each biometric measurement was estimated three times at each ultrasound scan and registered in the study database, in total 38,601 repeated measurements were included. Data was cleaned in several steps, described in detail in the publication of the individual biometric measurements ${ }^{29}$. In summary, data cleaning included identification of outliers, correction of erroneous data entry and deletion of unreasonable measurements.

Statistical analysis. Descriptive statistics were used for maternal characteristics at baseline, and for delivery and neonatal characteristics. Mean birthweight was compared in subgroups of the cohort based on country of birth and study site using an independent samples t-test for country of birth and a one-way ANOVA for study site, and $p$ value $<0.05$ was considered as statistically significant. The discrepancy between mean EFW and actual birthweight was compared using a paired samples t-test in women who delivered within two days after the last ultrasound scan.

Data from the biometric measurements was used to construct reference ranges for BPD, $\mathrm{HC}, \mathrm{AC}$ and $\mathrm{FL}^{29}$. The estimated fetal biometric measurements at each gestational day were used for estimating fetal weight using Hadlock's 3 rd formula ${ }^{19}$. In order to account for the violated normality assumptions of the data, the fetal growth measurements were log-transformed and modelled using a fixed and random effect multilevel regression methods $^{34}$. Fractional polynomial regression was first applied on the log transformed fetal measurement to define best fitting combination of fractional polynomials for the GA. To further illustrate the powers used, the fetal weight had a combination of 0.5 and 3 as the best fitting fractional polynomial powers. These parameters were then entered a fixed effect multilevel regression model to address the repeated measurements nature of the data reported for each fetus ${ }^{35,36}$. A two-level hierarchical model was sought for the repeated measurements of each fetus at each visit with a random effect for the identified fractional polynomial of GA and the intercept. The method of Johnsen et al. was employed to estimate the expected fetal measurements at each GA in weeks and compute their standard deviations (SD) and percentiles ${ }^{36}$.

In a sensitivity analysis, we excluded women with underweight $\left(\mathrm{BMI}<18.5 \mathrm{~kg} / \mathrm{m}^{2}\right)$ or obesity $(\mathrm{BMI} \geq 30.0 \mathrm{~kg} /$ $\mathrm{m}^{2}$ ) according to the World Health Organization (WHO) at first antenatal visit. We applied the same adjusted statistical models to the subset of the study cohort to estimate the expected fetal weight at each GA in weeks, and to compute the SD and percentiles. The reference ranges before and after exclusion of women with abnormal BMI were compared using an independent samples t-test, for all subjects as well as stratified according to offspring sex.

The median, $-2 \mathrm{SD}$ and $+2 \mathrm{SD}$ of the new reference ranges were compared with the corresponding values from the current Swedish reference, published by Maršál et $\mathrm{al}^{10}$. Further, the median, 2.5 th and 97.5 th percentiles were compared with the corresponding values from the Norwegian reference ranges more recently published by Johnsen et al. and the international standards published by the INTERGROWTH-21st project and WHO, respectively ${ }^{20,21,36}$.

Research involving human participants. The study was approved by the Regional Ethical Review Board in Uppsala (Nos. 2014/209 and 2014/209/2). All procedures involving human subjects were carried out in accordance with the ethical standards of the 1964 Helsinki declaration. All women participated voluntarily and gave their written informed consent. Any pregnancy complication recognized during the study was reported to the routine obstetric care at each study site and managed according to clinical practice.

\section{Results}

In total 2590 ultrasound scans were performed, of which 2551 were complete with data on BPD, HC, AC and FL and hence used for estimating fetal weight. The majority of the women, 526 of 583 (90\%), were scanned at least four times. In 187 women (32\%), the randomization protocol was completed with five scans evenly distributed over the second and third trimester of pregnancy. In 57 women (10\%), only two or three scans were performed. In 145 women (25\%), a scan was performed and registered outside the randomization protocol. These scans corresponded to the routine second trimester scan in 139 women, and the remaining 6 scans were performed in gestational week $22,26,30,32$, and 35 , respectively. The scans following the dating scan were fairly equally distributed over the GAs, see Fig. 1. There was a peak at week 18-19, corresponding to the routine ultrasound scan, and week 37-39. The mean dating discrepancy, i.e. the difference between estimated date of delivery according to BPD at first study visit and estimated date of delivery according to last menstrual period, was -0.1 days 


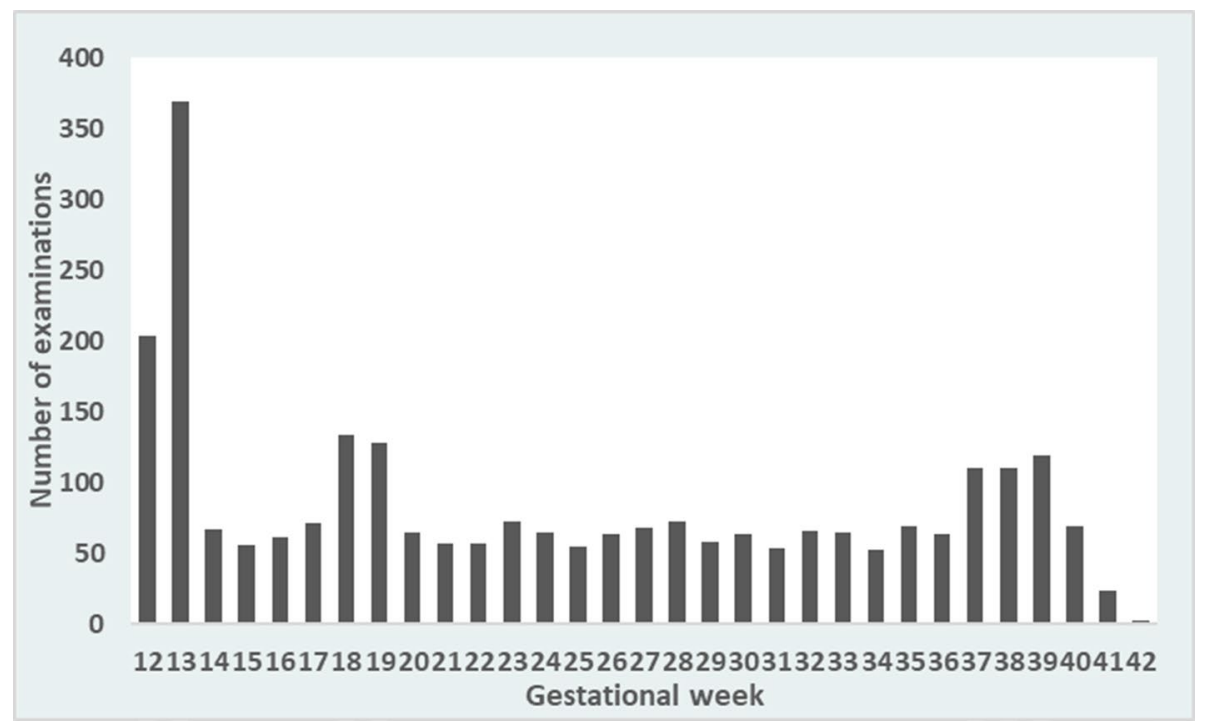

Figure 1. Distribution of ultrasound examinations by gestational age.

\begin{tabular}{|l|c|l|l|}
\hline Parameter & Median $(\mathrm{IQR})$ & Range & $\mathbf{n}(\%)$ \\
\hline Maternal age (years) & $29(26,33)$ & 19,44 & \\
\hline Maternal height $(\mathrm{cm})$ & $167(163,171)$ & 148,187 & \\
\hline Weight first visit $(\mathrm{kg})$ & $66(60,75)$ & 43,146 & \\
\hline Body mass index first visit $\left(\mathrm{kg} / \mathrm{m}^{2}\right)$ & $23.5(21,6,26,5)$ & $16.7,44.8$ & \\
\hline Nordic country of birth & & & $537(92.1 \%)$ \\
\hline Non-European country of birth & & & $34(5.8 \%)$ \\
\hline Smoking first visit & & & 0 \\
\hline Nulliparous & & & $250(42.9 \%)$ \\
\hline Gestational age at inclusion (days) & $92(90,94)$ & 87,101 & \\
\hline Gestational age at delivery (days) & $281(276,288)$ & 259,299 & \\
\hline Spontaneous vaginal delivery & & & $458(78.6 \%)$ \\
\hline Induction of labour & & & $40(6.9 \%)$ \\
\hline Newborn sex male & & & $308(52.8 \%)$ \\
\hline APGAR $<7$ at 5 min & & & $6(1.0 \%)$ \\
\hline Neonatal death & & & 0 \\
\hline Birthweight $(\mathrm{g})$ & $3625(3344,3925)$ & 2366,5100 & \\
\hline Birth length (cm) & $51(50,52)$ & 43,57 & \\
\hline
\end{tabular}

Table 1. Maternal and neonatal characteristics.

(SD 2.8 days) and the median discrepancy was 0 days. The dating discrepancy was slightly, but not statistically significantly larger for female than for male fetuses $(p=0.174)$; mean -0.5 days for females (SD 2.7 days) and 0.2 days for males (SD 2.7 days), respectively.

The median maternal age of the women at first study visit was 29 years. Body mass index (BMI) at first antenatal visit covered a range of $16.7-44.8 \mathrm{~kg} / \mathrm{m}^{2}$, with a median BMI of $23.5 \mathrm{~kg} / \mathrm{m}^{2}$. Fourteen women $(2.4 \%)$ were underweight $\left(\mathrm{BMI}<18.5 \mathrm{~kg} / \mathrm{m}^{2}\right), 145(24.9 \%)$ were overweight (BMI $\left.25.0-29.9 \mathrm{~kg} / \mathrm{m}^{2}\right)$, and $64(11.0 \%)$ were obese $\left(\mathrm{BMI}>30.0 \mathrm{~kg} / \mathrm{m}^{2}\right)$. The majority of the study population, $92 \%$, were born in a Nordic country (Sweden, Norway, Denmark, Finland or Iceland), and 5.8\% were of non-European origin. The median pregnancy duration at birth was 281 days.

Data on neonatal characteristics was available for 574 children (98.5\%), with median birthweight of $3625 \mathrm{~g}$ and median birth length of $51 \mathrm{~cm}$. The mean birthweight was $3645 \mathrm{~g}$. For children with a mother born in a Nordic country, the mean birthweight was $3652 \mathrm{~g}$, compared with $3563 \mathrm{~g}$ for children with a mother born in a non-Nordic country; a difference not statistically significant $(p=0.189)$. The mean birthweight was neither significantly different for children with a mother born in a European compared with a non-European country; $3650 \mathrm{~g}$ versus $3563 \mathrm{~g}(p=0.269)$. There was no statistically significant difference in mean birthweight between study sites; $3621 \mathrm{~g}$ in Uppsala, $3770 \mathrm{~g}$ in Falun, $3699 \mathrm{~g}$ in Katrineholm, $3606 \mathrm{~g}$ in Västerås, and $3591 \mathrm{~g}$ in Örebro, respectively $(p=0.051)$ Maternal and neonatal characteristics are summarized in Table 1. 


\begin{tabular}{|c|c|c|c|c|c|c|c|}
\hline GA (weeks ${ }^{\mathrm{a}}$ ) & $-3 \mathrm{SD}$ & $-2 S D$ & -1 SD & Median & $+1 \mathrm{SD}$ & $+2 S D$ & $+3 \mathrm{SD}$ \\
\hline 12 & 45 & 48 & 50 & 53 & 56 & 59 & 63 \\
\hline 13 & 59 & 62 & 66 & 69 & 74 & 78 & 82 \\
\hline 14 & 75 & 80 & 85 & 90 & 95 & 101 & 107 \\
\hline 15 & 95 & 101 & 108 & 115 & 122 & 129 & 138 \\
\hline 16 & 120 & 127 & 136 & 145 & 154 & 164 & 175 \\
\hline 17 & 149 & 159 & 169 & 181 & 193 & 206 & 220 \\
\hline 18 & 183 & 196 & 210 & 224 & 240 & 257 & 275 \\
\hline 19 & 223 & 240 & 257 & 276 & 296 & 317 & 340 \\
\hline 20 & 270 & 291 & 312 & 336 & 361 & 388 & 417 \\
\hline 21 & 324 & 350 & 377 & 406 & 437 & 471 & 508 \\
\hline 22 & 386 & 417 & 451 & 487 & 526 & 568 & 613 \\
\hline 23 & 457 & 495 & 535 & 579 & 627 & 678 & 734 \\
\hline 24 & 537 & 582 & 631 & 684 & 741 & 804 & 872 \\
\hline 25 & 626 & 679 & 738 & 802 & 871 & 946 & 1028 \\
\hline 26 & 724 & 788 & 858 & 933 & 1016 & 1105 & 1203 \\
\hline 27 & 832 & 907 & 989 & 1078 & 1176 & 1282 & 1398 \\
\hline 28 & 950 & 1038 & 1133 & 1238 & 1352 & 1476 & 1612 \\
\hline 29 & 1077 & 1178 & 1289 & 1410 & 1543 & 1688 & 1847 \\
\hline 30 & 1212 & 1328 & 1456 & 1596 & 1749 & 1917 & 2101 \\
\hline 31 & 1355 & \begin{tabular}{|l|}
1487 \\
\end{tabular} & 1633 & 1793 & 1969 & 2163 & 2375 \\
\hline 32 & \begin{tabular}{|l|}
1503 \\
\end{tabular} & 1654 & 1819 & 2002 & 2202 & 2423 & 2666 \\
\hline 33 & 1655 & 1825 & 2012 & 2218 & 2446 & 2697 & 2973 \\
\hline 34 & 1810 & 2000 & 2210 & 2442 & 2698 & 2981 & 3294 \\
\hline 35 & \begin{tabular}{|l|}
1964 \\
\end{tabular} & 2175 & 2409 & 2669 & 2956 & 3275 & 3628 \\
\hline 36 & 2114 & 2348 & 2608 & 2897 & 3218 & 3574 & 3970 \\
\hline 37 & 2257 & 2515 & 2802 & 3123 & 3479 & 3876 & 4319 \\
\hline 38 & 2391 & 2674 & 2989 & 3342 & 3736 & \begin{tabular}{|l|l}
4177 \\
\end{tabular} & \begin{tabular}{|l|l}
4670 \\
\end{tabular} \\
\hline 39 & 2512 & 2819 & 3164 & 3552 & 3986 & 4474 & 5021 \\
\hline 40 & 2616 & 2949 & 3324 & 3747 & 4224 & 4762 & 5367 \\
\hline 41 & 2701 & 3060 & 3466 & 3926 & 4447 & \begin{tabular}{|l|l|}
5037 \\
\end{tabular} & 5705 \\
\hline 42 & 2764 & 3148 & 3585 & \begin{tabular}{|l|l|}
4083 \\
\end{tabular} & \begin{tabular}{|l|}
4649 \\
\end{tabular} & 5295 & 6030 \\
\hline
\end{tabular}

Table 2. Medians and standard deviations (SD) of estimated fetal weight in grams by gestational age (GA) for male and female fetuses. ${ }^{a} \mathrm{GA}$ expressed as completed gestational weeks, e.g. 12 weeks corresponds to $12+0$ weeks or 84 gestational days.

The EFW using Hadlock's 3rd formula was compared with the actual birthweight in women who delivered within 2 days after the last ultrasound scan $(n=36)$. The difference between the mean EFW and actual birthweight was $13 \mathrm{~g}(p=0.798)$.

The median and variance of EFW by GA for male and female fetuses from gestational week 12 until gestational week 42 are shown in Tables 2 and 3 and Eq. (1). The variance is expressed in standard deviations $(+3$ SD,+2 SD, +1 SD, median, -1 SD, -2 SD and -3 SD) in Table 2 and in percentiles (2.5th, 5th, 10th, 25th, median, 75th, 90th, 95th and 97.5th) in Table 3.

Equation 1. Mean and variance for estimated fetal weight in male and female fetuses.

$$
\begin{aligned}
E\left(Z_{\mathrm{i}}\right)= & -2.80+\left[1.97 \mathrm{GA}_{\mathrm{i}}^{0.5}\right]+\left[-0.00002 \mathrm{GA}_{\mathrm{i}}^{3}\right] \\
\operatorname{Var}\left(Z_{\mathrm{i}}\right)= & 0.02+\left[-0.002 \mathrm{GA}_{\mathrm{i}}\right]+\left[-0.01 \mathrm{GA}_{\mathrm{i}}^{0.5}\right]+\left[2.39 \mathrm{e}-07 \mathrm{GA}_{\mathrm{i}}^{3}\right] \\
& +\left[-9.92 \mathrm{e}-08 \mathrm{GA}_{\mathrm{i}}^{0.5} \mathrm{GA}_{\mathrm{i}}^{3}\right]+\left[4.12 \mathrm{e}-12 \mathrm{GA}_{\mathrm{i}}^{6}\right]
\end{aligned}
$$

Figure 2 shows the median and variance in SD of EFW by GA from gestational day 84 (gestational week 12+0) until gestational day 294 (gestational week $42+0$ ). Supplementary table 1a shows the medians and variance in SD of EFW by GA for male and female fetuses for each day of the pregnancy from gestational day 84 until gestational day 294 .

Figure 3 shows the median and variance in percentiles of EFW by GA from gestational day 84 until gestational day 294. Supplementary table $1 \mathrm{~b}$ shows the medians and variance in percentiles of EFW by GA for male and female fetuses for each day of the pregnancy from gestational day 84 until gestational day 294 .

Figure 4 shows the sex specific reference ranges for EFW by GA at 2.5 th, 50 th and 97.5 th percentiles. Supplementary tables $2 a-b$ show the medians and variance in SD (2a) and percentiles (2b) of EFW by GA for male 


\begin{tabular}{|c|c|c|c|c|c|c|c|c|c|}
\hline GA (weeks $\left.{ }^{a}\right)$ & 2.5th & 5th & 10th & 25th & Median & 75th & 90th & 95th & 97.5th \\
\hline 12 & 48 & 49 & 50 & 51 & 53 & 55 & 57 & 58 & 59 \\
\hline 13 & 62 & 63 & 65 & 67 & 69 & 72 & 75 & 76 & 78 \\
\hline 14 & 80 & 81 & 83 & 86 & 90 & 93 & 97 & 99 & 101 \\
\hline 15 & 102 & 104 & 106 & 110 & 115 & 119 & 124 & 127 & 129 \\
\hline 16 & 128 & 130 & 133 & 139 & 145 & 151 & 157 & 161 & 164 \\
\hline 17 & 159 & 162 & 166 & 173 & 181 & 189 & 197 & 202 & 206 \\
\hline 18 & 196 & 201 & 206 & 214 & 224 & 235 & 245 & 251 & 256 \\
\hline 19 & 240 & 246 & 252 & 263 & 276 & 289 & 302 & 309 & 316 \\
\hline 20 & 291 & 298 & 306 & 320 & 336 & 353 & 369 & 378 & 387 \\
\hline 21 & 351 & 359 & 369 & 386 & 406 & 427 & 447 & 459 & 470 \\
\hline 22 & 419 & 429 & 441 & 462 & 487 & 513 & 537 & 552 & 566 \\
\hline 23 & 496 & 509 & 523 & 549 & 579 & 611 & 641 & 659 & 676 \\
\hline 24 & 584 & 599 & 617 & 648 & 684 & 722 & 759 & 781 & 801 \\
\hline 25 & 682 & 700 & 721 & 758 & 802 & 848 & 892 & 919 & 943 \\
\hline 26 & 791 & 812 & 837 & 882 & 933 & 988 & 1040 & 1073 & 1102 \\
\hline 27 & 911 & 936 & 965 & 1017 & \begin{tabular}{|l}
1078 \\
\end{tabular} & 1143 & 1205 & 1243 & 1277 \\
\hline 28 & 1041 & 1071 & 1105 & 1166 & 1238 & 1313 & 1386 & 1431 & 1471 \\
\hline 29 & 1182 & 1216 & 1257 & 1327 & 1410 & 1498 & 1583 & 1635 & 1682 \\
\hline 30 & 1333 & 1372 & 1419 & 1500 & 1596 & 1698 & 1795 & 1856 & 1910 \\
\hline 31 & 1493 & 1538 & \begin{tabular}{|l|}
1591 \\
\end{tabular} & 1684 & 1793 & 1910 & 2022 & 2092 & 2154 \\
\hline 32 & 1660 & 1711 & \begin{tabular}{|l}
1771 \\
\end{tabular} & \begin{tabular}{|l|l}
1877 \\
\end{tabular} & 2002 & 2135 & 2262 & 2342 & 2414 \\
\hline 33 & 1832 & \begin{tabular}{|l|l|}
1889 \\
\end{tabular} & \begin{tabular}{|l|l|}
1958 \\
\end{tabular} & 2077 & 2218 & 2369 & 2514 & 2605 & 2686 \\
\hline 34 & 2008 & 2072 & 2148 & 2283 & 2442 & 2612 & 2775 & 2878 & 2970 \\
\hline 35 & 2184 & 2255 & 2341 & 2491 & 2669 & 2859 & 3043 & 3158 & 3262 \\
\hline 36 & 2358 & 2437 & 2532 & 2699 & 2897 & 3110 & 3315 & 3444 & 3559 \\
\hline 37 & 2526 & 2614 & 2718 & 2903 & 3123 & 3359 & 3587 & 3730 & 3860 \\
\hline 38 & 2686 & 2782 & 2897 & 3100 & 3342 & 3603 & 3856 & 4015 & \begin{tabular}{|l|l|}
4159 \\
\end{tabular} \\
\hline 39 & 2832 & 2937 & 3063 & 3286 & 3552 & 3839 & 4118 & 4294 & 4453 \\
\hline 40 & 2963 & 3077 & 3214 & 3457 & 3747 & 4062 & 4369 & 4563 & 4739 \\
\hline 41 & 3075 & 3198 & 3346 & 3609 & 3926 & 4270 & 4606 & 4819 & 5011 \\
\hline 42 & 3164 & 3297 & 3456 & \begin{tabular}{|l|l|}
3740 \\
\end{tabular} & 4083 & 4456 & 4823 & 5056 & 5267 \\
\hline
\end{tabular}

Table 3. Medians and percentiles of estimated fetal weight in grams by gestational age (GA) for male and female fetuses. ${ }^{\text {a }} \mathrm{GA}$ expressed as completed gestational weeks, e.g. 12 weeks corresponds to $12+0$ weeks or 84 gestational days.

fetuses. Supplementary tables 3a-b show the medians and variance in SD (3a) and percentiles (3b) of EFW by GA for female fetuses. All supplemental tables enclose the full equations of the median and variance for EFW.

Figure 5 shows the distribution of the raw data with fitted percentiles for estimated fetal weight by GA.

In comparison with the currently used Swedish growth reference ranges by Maršál et $\mathrm{al}^{10}$, the new reference ranges had comparable median EFW until gestational week 33, see Fig. 6a. Thereafter, the differences increased with advancing GA, with higher median EFW in the new reference ranges. From gestational week 25 to 33 , the difference in median EFW was less than 2\%. In week 39, the difference in median EFW was the largest; $4.4 \%$, corresponding to $158 \mathrm{~g}$. For +2 SD of EFW, the difference exceeded 5\% from week 38, and was the highest in week $42 ; 7.9 \%$, corresponding to $419 \mathrm{~g}$. Before gestational week $33,+2$ SD of EFW was lower in the new reference ranges. In the new reference ranges, -2 SD corresponded to approximately $10 \%$ higher EFW from week 25 to 39 , thereafter the differences declined, but -2 SD of EFW remained higher in the new reference ranges throughout pregnancy. In week 38, the difference was $244 \mathrm{~g}$, or $9.1 \%$, and in week $41,205 \mathrm{~g}$, or $7.0 \%$.

Next, the new reference ranges were compared with the Norwegian reference ranges by Johnsen et al. from $2006^{36}$, the standards from the INTERGROWTH-21st project ${ }^{21}$ and $\mathrm{WHO}^{20}$, see Fig. $6 \mathrm{~b}-\mathrm{d}$. Compared with the Norwegian reference ranges, the new reference ranges had lower median EFW until week 31, and thereafter higher median EFW, see Fig. 6b. In the new reference ranges, the 2.5 th percentile was higher in all gestational weeks, with the most pronounced differences in late pregnancy. Compared with the INTERGROWTH-21st project, the median, the 2.5th, and the 97.5th percentiles of EFW were higher in the new reference ranges from gestational week 24 and onwards, see Fig. 6c. The new reference ranges had higher median EFW also in comparison with WHO; 3.6-5.3\% from gestational week 27 and forward. In the new reference ranges, the 2.5th percentile was higher throughout pregnancy, declining from $12.7 \%$ in week 15 , to $10.4 \%$ in week 36 , the later corresponding to $245 \mathrm{~g}$. Thereafter, the difference was below $10 \%$. The 97.5 th percentile was lower in the new reference ranges in early pregnancy, but the curves crossed in week 29 , with a maximum of $4.7 \%$, or $224 \mathrm{~g}$, in week 40 . 


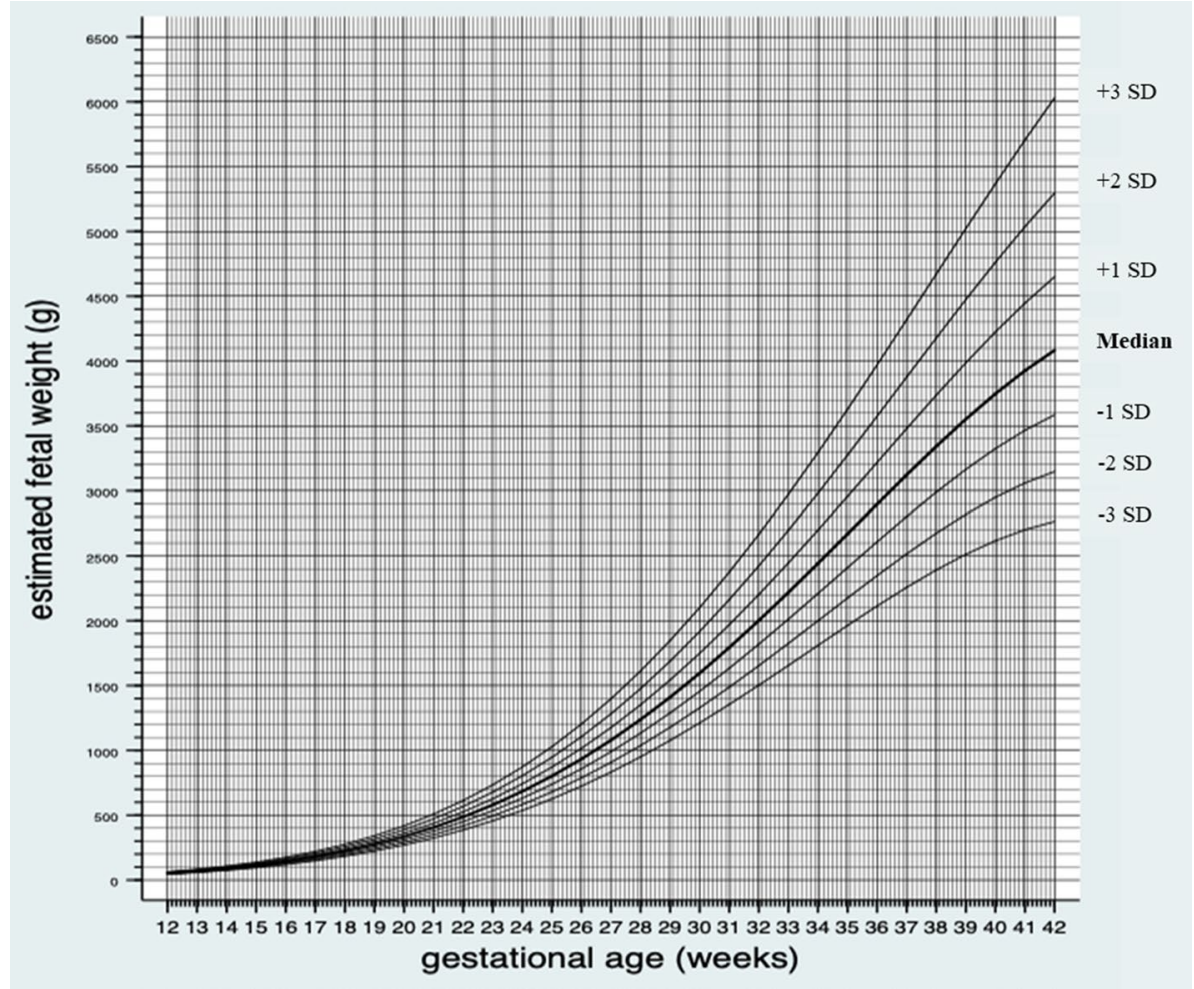

Figure 2. Median and variance in standard deviations (SD) $(+3 \mathrm{SD},+2 \mathrm{SD},+1 \mathrm{SD}$, median, $-1 \mathrm{SD},-2 \mathrm{SD}$ and $-3 \mathrm{SD}$ ) of estimated fetal weight in grams ( $\mathrm{g}$ ) by gestational age for male and female fetuses.

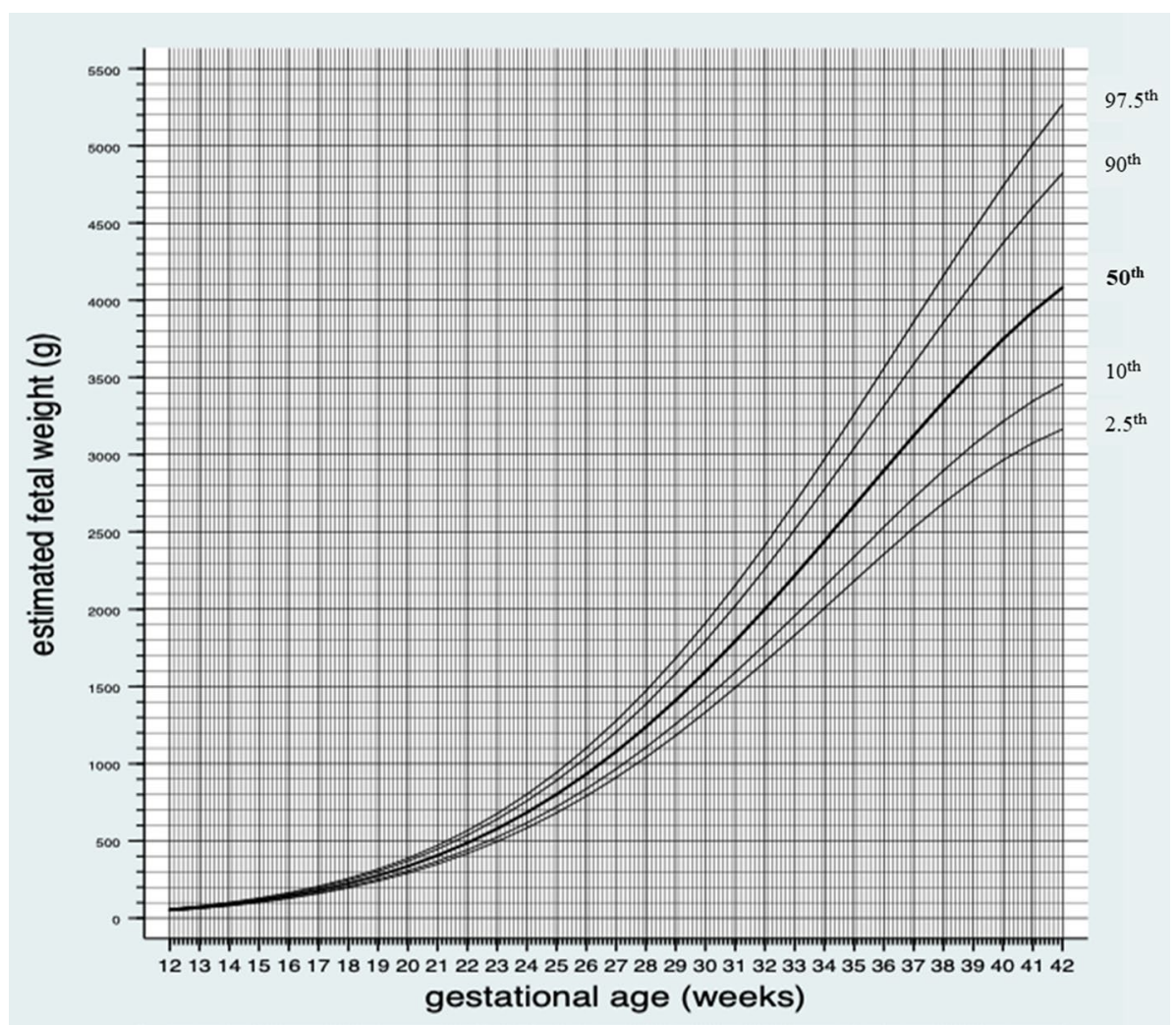

Figure 3. Median and variance in percentiles (2.5th, 10th, 50th, 90th and 97.5th) of estimated fetal weight by gestational age for male and female fetuses. 


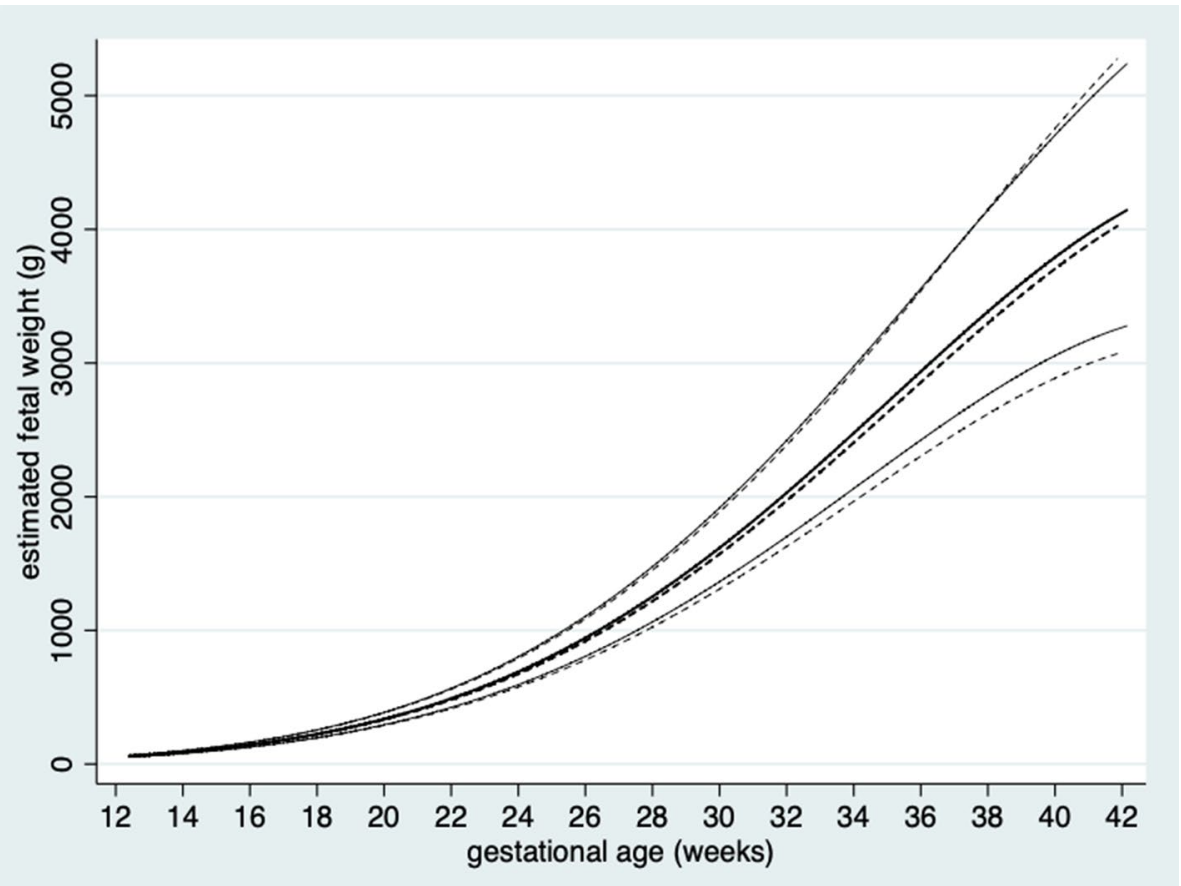

Figure 4. Sex specific reference ranges for estimated fetal weight by gestational age at 2.5 th, 50th and 97.5 th percentiles for male fetuses (continuous) and female fetuses (dashed).

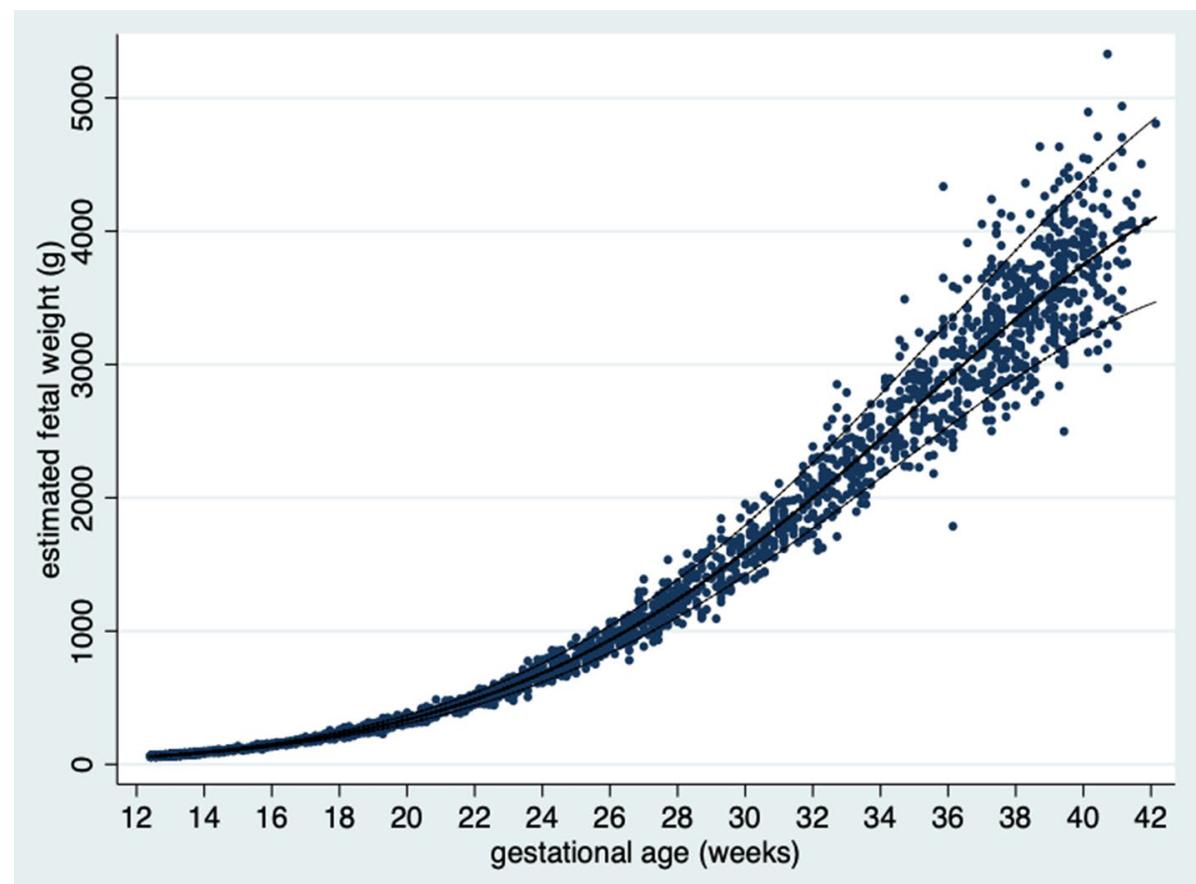

Figure 5. Raw data $(n=2551)$ with fitted percentiles $(10$ th, 50 th, 90 th $)$ for estimated fetal weight by gestational age.

When comparing women with BMI $18.5-29.9 \mathrm{~kg} / \mathrm{m}^{2}$ in early pregnancy to the whole study population, there were no statistically significant differences in median EFW ( $p=0.991$ for both sexes, $p=0.985$ for males and $p=0.998$ for females). The difference in median EFW was the largest in week 42 (14 g). Supplementary table $4 \mathrm{a}-\mathrm{b}$ show the medians and variance in SD (4a) and percentiles (4b) of EFW by GA for male and female fetuses in the subset of women with BMI $18.5-29.9 \mathrm{~kg} / \mathrm{m}^{2}$. 

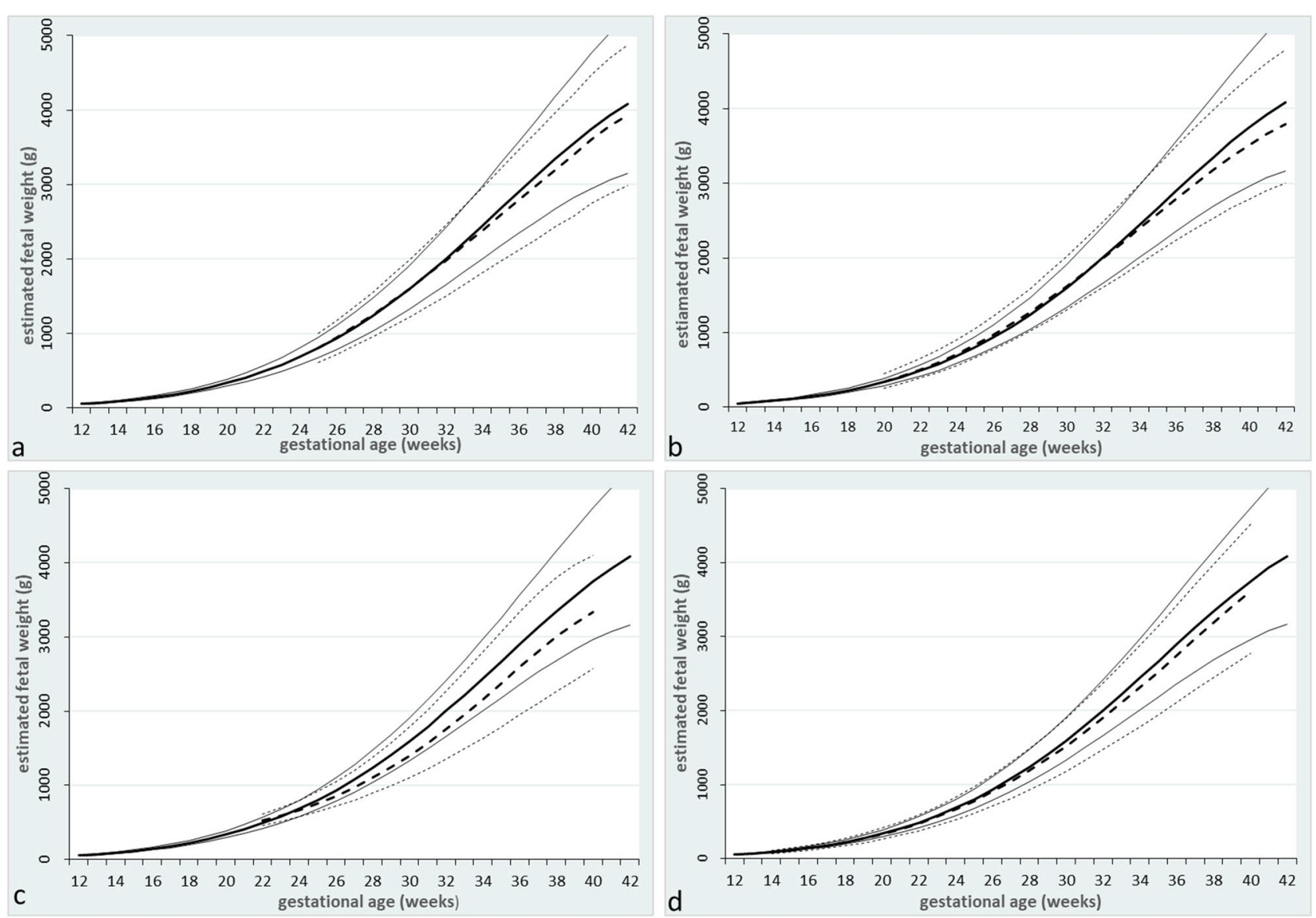

Figure 6. Comparisons of estimated fetal weight for males and females in the present study with other reference ranges; (a) new reference ranges (continuous) versus Maršál et al ${ }^{10}$ (dashed), $+2 \mathrm{SD}$, median, -2 $\mathrm{SD}$; (b) new reference ranges (continuous) versus Johnsen et $\mathrm{al}^{36}$ (dashed), 2.5th percentile, median, 97.5th percentile; (c) new reference ranges (continuous) versus INTERGROWTH-21st ${ }^{21}$ (dashed), 2.5th percentile, median, 97.5th percentile; (d) new reference ranges (continuous) versus $\mathrm{WHO}^{20}$ (dashed), 2.5th percentile, median, 97.5 th percentile.

\section{Discussion}

In this study we present new Swedish reference ranges of EFW by GA to be used for assessing fetal weight and growth from gestational week 12 to 42 .

A strength of the study is the adherence to a recommended study design for construction of reference ranges of fetal size and growth ${ }^{7,8,37}$. Large efforts have been made to ensure that the study population only includes pregnancies with low risk of aberrant fetal growth. Another strength is the prospective enrolment of study subjects and the use of modern statistical methods, which take into account the repeated measurements of each fetus and increased variation of EFW with advancing GA. The study design and statistical methods allow the growth reference ranges to be used to reliably evaluate EFW, both regarding size and growth. Moreover, the scans were performed by a limited number of experienced sonographers, and the quality of the original data was high, with a low rate of erroneous data ${ }^{29}$.

Another major strength of the study is the distribution of ultrasound scans throughout the entire second and third trimester. This is particularly valuable in comparison with the currently used Swedish growth reference ranges by Maršál et al., which do not include any intrauterine measurements before gestational week $25^{10}$. Assessment of EFW is an important part of obstetric care, even before the limit of extrauterine viability, practically considered as gestational week $22+0$ in Sweden today. If the new reference ranges are adopted in clinical practice, assessment of EFW and postnatal growth in the extreme preterm period will be compared with reference ranges based on observational data rather than the currently used extrapolated data.

A limitation of the study is the homogeneity of the study population. In order to recruit a study cohort with similar ethnic background as the Swedish pregnant population, the primary care centres that recruited study subjects were singled out with great care. Several of the centres were situated in areas with a large immigrated population. Further efforts were made to recruit study subjects of non-Nordic origin by offering study information not only in Swedish, but also in English and Arabic. However, less than $10 \%$ of the study subjects were born outside the Nordic countries, compared with $28.5 \%$ among Swedish pregnant women $2018^{38}$. The sampling bias towards homogenous ethnicity was probably unavoidable in part due to language difficulties, as interpreters could 
not be offered at all study visits due to limited financial means. Additionally, a higher percentage of the women born outside the Nordic countries were excluded from the study due to pregnancy complications; 15 versus $10 \%$.

We included women regardless of their BMI in early pregnancy, despite the increased risks of restricted as well as exaggerated fetal growth associated with low and high BMI. The rationale behind this decision was that we aimed to recruit a study population similar to the current healthy pregnant population in Sweden. Mean maternal age and BMI were similar to those in the current Swedish pregnant population, and thus the study population can be seen as representative for Swedish pregnant women ${ }^{38}$. The vast majority of Swedish women of fertile age are well nourished, and low BMI is rarely a sign of undernutrition. However, the inclusion of women with obesity might affect the results in a way that skews the growth reference ranges towards a higher EFW, which might lead to an underestimation of the number of fetuses suspected as large for gestational age (LGA) during pregnancy. In that aspect, the inclusion of obese women is a limitation. The increased risk of pregnancy complications in women with increased BMI is reflected in the higher rate of exclusion due to pregnancy complications in overweight and obese women. However, in a sensitivity analysis, the growth reference ranges were compared with and without the inclusion of women with BMI less than $18.5 \mathrm{~kg} / \mathrm{m}^{2}$ or more than $30 \mathrm{~kg} / \mathrm{m}^{2}$. The difference in median EFW, as well as for -2 SD and +2 SD, was clinically insignificant if women with abnormal BMI were excluded. Therefore, we suggest that the results from the complete study population should be used, since the larger cohort is a strength in later GAs, as the variation in fetal weight increases and is better evaluated with a larger cohort. Moreover, the inclusion of women regardless of BMI entails a higher generalisability to the background population.

The mean birthweight in this study was approximately $150 \mathrm{~g}$ higher than the mean birthweight for all children born in Sweden in 2018; 3646 versus $3497 \mathrm{~g}^{38}$. In contrast to national data, the study cohort only includes children born term and of women without conditions that affect fetal growth. The mean birthweight can therefore be regarded as representative for Swedish term born children.

In clinical practice, there is a large variety of formulae used for estimating fetal weight. In this study, we used Hadlock et al.s third formula for EFW, which includes the biometric measurements BPD, HC, AC and FL. The formula for EFW was chosen based on compelling evidence from repeated studies which have proven Hadlock et al.s formulae to be superior or non-inferior to a variety of frequently used or more recently developed EFW formulae ${ }^{15-18,39,40}$. In Sweden, the formula adapted by Persson and Weldner in 1986 is recommended by the Swedish Society of Obstetrics and Gynecology and commonly used for estimation of fetal weight ${ }^{30,41}$. Since the Persson Weldner formula was not included in any of the cited studies, its accuracy is not evaluated in comparison with other formulae for EFW. Moreover, the accuracy of the Person Weldner formula has not been assessed in early gestational weeks. The different formulae presented by Hadlock et al. include various biometric measurements, but their performance is largely comparable whether BPD is included or not ${ }^{15}$. In the study, we have only estimated fetal weight if HC and BPD were present. The use of Hadlock's third formula in our study seems appropriate, since the difference between mean EFW and actual birthweight was negligible in women who delivered within two days from the last ultrasound scan.

Over the years, a multitude of national and international standards of EFW have been presented. Despite the large efforts made during the last decade to create a standard that can be universally adopted, doubts have been raised regarding the notion of "one size fits all"20,22-27. Vieira et al. compared the proportion of children classified as small for gestational age (SGA) and LGA at birth using three different growth reference ranges in a Swedish cohort ${ }^{42}$. The standard presented by the INTERGROWTH-21st project only classified $3.1 \%$ as below the 10th percentile for GA and sex. In concordance with these findings, the difficulty in implementing international standards have repeatedly been highlighted since the sensitivity and specificity in detecting growth restricted fetuses or fetuses with adverse perinatal outcome vary widely in different populations ${ }^{12,43-46}$. Thus, great care must be taken in choosing a national reference for fetal size and growth.

Compared with the currently used Swedish growth reference ranges by Maršál et al., the EFW was considerably higher in our study, especially in late pregnancy. The higher EFW in our study might in part be explained by the stricter inclusion criteria, e.g. women who smoked and had other risk factors for fetal growth restriction were not eligible. Further, there were large differences between our and Maršál et al.'s reference ranges at all GAs for the EFW at - 2 SD, which is used as cut-off for SGA in Sweden. The larger span in late GAs between +2 and -2 SD in our reference ranges may be a reflection of how a larger study sample and modern statistical methods estimate the increasing variation in fetal weight with increased GA in a correct way.

The pregnant populations in the Nordic countries are quite homogeneous, with similar mean birthweight for live born children, ranging from $3484 \mathrm{~g}$ in Denmark to $3501 \mathrm{~g}$ in Sweden, and the somewhat higher Icelandic mean of $3592 \mathrm{~g}^{47}$. We compared our reference ranges with the most recently published Nordic reference ranges by Johnsen et $\mathrm{al}^{36}$. The higher median EFW in our reference ranges is most likely explained by the fact that Johnsen et al. did not exclude women with pregnancy complications from the analyses, rather than by differences in the populations. The reference ranges created by INTERGROWTH-21st project and the WHO on the other hand are based on populations that are quite different from the Swedish pregnant population and our study population, with shorter maternal height $(162 \mathrm{~cm}$ vs $167 \mathrm{~cm})$, higher rate of nulliparous study subjects (68\% vs $43 \%)$ and lower mean birthweight ( $3.3 \mathrm{~kg}$ vs $3.6 \mathrm{~kg}$ ). We believe that optimal fetal weight is underestimated if these international reference ranges are used in the Nordic populations, but further studies are needed to confirm this speculation.

The distribution of percentiles in a growth reference range will affect the percentage of fetuses that are considered SGA or LGA. Hence, if the birthweight percentiles in the population are significantly higher than the percentiles of the reference range used, SGA fetuses are expected to be misclassified as appropriate for GA. Accordingly, using the presently used reference ranges, their increased risk of adverse perinatal outcome is most probably overseen. Likewise, if the mean birthweight is lower than the 50th percentile of the reference range, an increased number of fetuses are falsely suspected as SGA, and thereby risk unnecessary obstetric interventions 
with increased risk of adverse perinatal outcome ${ }^{48}$. If these new reference ranges with higher EFW percentiles during the third trimester are adopted in clinical practice, the risk of missing to identify growth restricted fetuses might become lower in comparison to using the current growth reference ranges or standards created by INTERGROWTH-21st project or the WHO. Likewise, the risk of misclassifying fetuses as LGA might be lower with our new reference ranges, and hence the risk of unnecessary interventions should be reduced. Further studies are needed to evaluate the sensitivity and specificity of the new growth reference ranges to detect SGA fetuses, and to assess appropriate cut-offs for SGA and LGA in order to identify fetuses at high risk of adverse perinatal outcome, both from an obstetric and neonatal perspective.

\section{Data availability}

The datasets generated during and/or analyzed during the current study are not publicly available due to the ethical and legal restrictions prohibiting the sharing of personal data, but are available from the corresponding author on reasonable request.

Received: 24 March 2021; Accepted: 2 June 2021

Published online: 14 June 2021

\section{References}

1. Gardosi, J., Madurasinghe, V., Williams, M., Malik, A. \& Francis, A. Maternal and fetal risk factors for stillbirth: population based study. BMJ (Clin. Res. Ed.) 346, f108. https://doi.org/10.1136/bmj.f108 (2013).

2. Cnattingius, S. The small-for-gestational-age infant: obstetrical management and perinatal outcome. Ups. J. Med. Sci. 94, 55-65. https://doi.org/10.3109/03009738909179247 (1989).

3. Baschat, A. A. et al. Predictors of neonatal outcome in early-onset placental dysfunction. Obstet. Gynecol. 109, 253-261. https:// doi.org/10.1097/01.aog.0000253215.79121.75 (2007).

4. Bergman, E., Kieler, H., Petzold, M. G., Sonesson, C. \& Axelsson, O. Symphysis-fundus measurements for detection of small for gestational age pregnancies. Acta Obstet. Gynecol. Scand. 85, 407-412. https://doi.org/10.1080/00016340500432390 (2006).

5. American College of Obstetricians and Gynecologists. ACOG Practice Bulletin No. 204: Fetal Growth Restriction. Obstet. Gynecol. 133, e97-e109. https://doi.org/10.1097/aog.0000000000003070 (2019).

6. Royal College of Obstetricians and Gynaecologists. The investigation and management of the small-for-gestational age fetus. Green-top Guideline No. 31. 2nd edn. (2014).

7. Altman, D. G. \& Chitty, L. S. Design and analysis of studies to derive charts of fetal size. Ultrasound Obstet. Gynecol. 3, 378-384. https://doi.org/10.1046/j.1469-0705.1993.03060378.x (1993).

8. Ohuma, E. O. \& Altman, D. G. Design and other methodological considerations for the construction of human fetal and neonatal size and growth charts. Stat. Med. https://doi.org/10.1002/sim.8000 (2018).

9. Zeitlin, J., Ancel, P. Y., Saurel-Cubizolles, M. J. \& Papiernik, E. The relationship between intrauterine growth restriction and preterm delivery: an empirical approach using data from a European case-control study. BJOG Int. J. Obstet. Gynaecol. 107, 750-758. https://doi.org/10.1111/j.1471-0528.2000.tb13336.x (2000).

10. Marsal, K. et al. Intrauterine growth curves based on ultrasonically estimated foetal weights. Acta Paediatr. 85, 843-848 (1996).

11. Salomon, L. J., Bernard, J. P. \& Ville, Y. Estimation of fetal weight: reference range at 20-36 weeks' gestation and comparison with actual birth-weight reference range. Ultrasound Obstet. Gynecol. 29, 550-555. https://doi.org/10.1002/uog.4019 (2007).

12. Nicolaides, K. H., Wright, D., Syngelaki, A., Wright, A. \& Akolekar, R. Fetal Medicine Foundation fetal and neonatal population weight charts. Ultrasound Obstet. Gynecol. 52, 44-51. https://doi.org/10.1002/uog.19073 (2018).

13. Bhatia, J. Growth curves: how to best measure growth of the preterm infant. J. Pediatr. 162, S2-S6. https://doi.org/10.1016/j.jpeds. 2012.11.047 (2013).

14. Kiserud, T. \& Johnsen, S. L. Biometric assessment. Best Pract. Res. Clin. Obstet. Gynaecol. 23, 819-831. https://doi.org/10.1016/j. bpobgyn.2009.06.007 (2009).

15. Hammami, A., Mazer Zumaeta, A., Syngelaki, A., Akolekar, R. \& Nicolaides, K. H. Ultrasonographic estimation of fetal weight: development of new model and assessment of performance of previous models. Ultrasound Obstet. Gynecol. 52, 35-43. https:// doi.org/10.1002/uog.19066 (2018).

16. Kong, C. W. \& To, W. W. K. Comparison of the accuracy of INTERGROWTH-21 formula with other ultrasound formulae in fetal weight estimation. Taiwan J. Obstet. Gynecol. 58, 273-277. https://doi.org/10.1016/j.tjog.2019.01.019 (2019).

17. Blue, N. R. et al. The Hadlock method is superior to newer methods for the prediction of the birth weight percentile. J. Ultrasound Med. 38, 587-596. https://doi.org/10.1002/jum.14725 (2019).

18. Monier, I. et al. Comparison of the Hadlock and INTERGROWTH formulas for calculating estimated fetal weight in a preterm population in France. Am. J. Obstet. Gynecol. 219, 476 e471-476 e412. https://doi.org/10.1016/j.ajog.2018.08.012 (2018).

19. Hadlock, F. P., Harrist, R. B., Sharman, R. S., Deter, R. L. \& Park, S. K. Estimation of fetal weight with the use of head, body, and femur measurements—a prospective study. Am. J. Obstet. Gynecol. 151, 333-337 (1985).

20. Kiserud, T. et al. The World Health Organization Fetal Growth Charts: a multinational longitudinal study of ultrasound biometric measurements and estimated fetal weight. PLoS Med. 14, e1002220. https://doi.org/10.1371/journal.pmed.1002220 (2017).

21. Stirnemann, J. et al. International estimated fetal weight standards of the INTERGROWTH-21(st) Project. Ultrasound Obstet. Gynecol. 49, 478-486. https://doi.org/10.1002/uog.17347 (2017).

22. Kierans, W. J. et al. Does one size fit all? The case for ethnic-specific standards of fetal growth. BMC Pregnancy Childbirth $8,1$. https://doi.org/10.1186/1471-2393-8-1 (2008).

23. Hanley, G. E. \& Janssen, P. A. Ethnicity-specific birthweight distributions improve identification of term newborns at risk for short-term morbidity. Am. J. Obstet. Gynecol. 209(428), e421-e426. https://doi.org/10.1016/j.ajog.2013.06.042 (2013).

24. Sletner, L., Kiserud, T., Vangen, S., Nakstad, B. \& Jenum, A. K. Effects of applying universal fetal growth standards in a Scandinavian multi-ethnic population. Acta Obstet. Gynecol. Scand. 97, 168-179. https://doi.org/10.1111/aogs.13269 (2018).

25. Grantz, K. L., Hediger, M. L., Liu, D. \& Buck Louis, G. M. Fetal growth standards: the NICHD fetal growth study approach in context with INTERGROWTH-21st and the World Health Organization Multicentre Growth Reference Study. Am. J. Obstet. Gynecol. 218, S641-S655. https://doi.org/10.1016/j.ajog.2017.11.593 (2018) (e628).

26. Kabiri, D. et al. Prediction of adverse perinatal outcome by fetal biometry: comparison of customized and population-based standards. Ultrasound Obstet. Gynecol. 55, 177-188. https://doi.org/10.1002/uog.20299 (2020).

27. Cheng, Y. K. Y., Lu, J., Leung, T. Y., Chan, Y. M. \& Sahota, D. S. Prospective assessment of INTERGROWTH-21(st) and World Health Organization estimated fetal weight reference curves. Ultrasound Obstet. Gynecol. 51, 792-798. https://doi.org/10.1002/ uog.17514 (2018).

28. Salomon, L. J. et al. Practice guidelines for performance of the routine mid-trimester fetal ultrasound scan. Ultrasound Obstet. Gynecol. 37, 116-126. https://doi.org/10.1002/uog.8831 (2011). 
29. Lindström, L. et al. Swedish intrauterine growth reference ranges of biometric measurements of fetal head, abdomen and femur. Sci. Rep. 10, 22441. https://doi.org/10.1038/s41598-020-79797-8 (2020).

30. Rekommendationer för fetometri. (Swedish Society of Obstetrics and Gynecology, https://www.sfog.se/media/98796/biometri_ 2011_sep_29_rek.pdf, 2011). (Accepted on 27 May 2021).

31. Selbing, A. \& Kjessler, B. Conceptual dating by ultrasonic measurement of the fetal biparietal diameter in early pregnancy. Acta Obstet. Gynecol. Scand. 64, 593-597. https://doi.org/10.3109/00016348509156368 (1985).

32. Saltvedt, S. et al. Ultrasound dating at 12-14 or 15-20 weeks of gestation? A prospective cross-validation of established dating formulae in a population of in-vitro fertilized pregnancies randomized to early or late dating scan. Ultrasound Obstet. Gynecol. 24, 42-50. https://doi.org/10.1002/uog.1047 (2004).

33. Salomon, L. J. et al. ISUOG practice guidelines: ultrasound assessment of fetal biometry and growth. Ultrasound Obstet. Gynecol. 53, 715-723. https://doi.org/10.1002/uog.20272 (2019).

34. Ohuma, E. O. \& Altman, D. G. Statistical methodology for constructing gestational age-related charts using cross-sectional and longitudinal data: the INTERGROWTH-21(st) project as a case study. Stat. Med. 38, 3507-3526. https://doi.org/10.1002/sim.8018 (2019).

35. Johnsen, S. L., Wilsgaard, T., Rasmussen, S., Sollien, R. \& Kiserud, T. Longitudinal reference charts for growth of the fetal head, abdomen and femur. Eur. J. Obstet. Gynecol. Reprod. Biol. 127, 172-185. https://doi.org/10.1016/j.ejogrb.2005.10.004 (2006).

36. Johnsen, S. L., Rasmussen, S., Wilsgaard, T., Sollien, R. \& Kiserud, T. Longitudinal reference ranges for estimated fetal weight. Acta Obstet. Gynecol. Scand. 85, 286-297. https://doi.org/10.1080/00016340600569133 (2006).

37. Ioannou, C. et al. Systematic review of methodology used in ultrasound studies aimed at creating charts of fetal size. BJOG 119, 1425-1439. https://doi.org/10.1111/j.1471-0528.2012.03451.x (2012).

38. Statistics on Pregnancies, Deliveries and Newborn Infants 2018. (National Board of Health and Welfare. https://www.socialstyr elsen.se/globalassets/sharepoint-dokument/artikelkatalog/statistik/2020-2-6623.pdf, 2020). (Accepted on 27 May 2021).

39. Sovio, U. \& Smith, G. C. S. Comparison of estimated fetal weight percentiles near term for predicting extremes of birth weight percentile. Am. J. Obstet. Gynecol. https://doi.org/10.1016/j.ajog.2020.08.054 (2020).

40. Esinler, D. et al. Finding the best formula to predict the fetal weight: comparison of 18 formulas. Gynecol. Obstet. Invest. 80, 78-84. https://doi.org/10.1159/000365814 (2015).

41. Persson, P. H. \& Weldner, B. M. Intra-uterine weight curves obtained by ultrasound. Acta Obstet. Gynecol. Scand. 65, 169-173. https://doi.org/10.3109/00016348609158374 (1986).

42. Vieira, M. C., Relph, S., Persson, M., Seed, P. T. \& Pasupathy, D. Determination of birth-weight centile thresholds associated with adverse perinatal outcomes using population, customised, and Intergrowth charts: a Swedish population-based cohort study. PLoS Med. 16, e1002902. https://doi.org/10.1371/journal.pmed.1002902 (2019).

43. Anderson, N. H., Sadler, L. C., McKinlay, C. J. D. \& McCowan, L. M. E. INTERGROWTH-21st vs customized birthweight standards for identification of perinatal mortality and morbidity. Am. J. Obstet. Gynecol. 214, 509 e501-509 e507. https://doi.org/10.1016/j. ajog.2015.10.931 (2016).

44. Nwabuobi, C. et al. Comparing INTERGROWTH-21st Century and Hadlock growth standards to predict small for gestational age and short-term neonatal outcomes. J. Matern. Fetal Neonatal Med. https://doi.org/10.1080/14767058.2018.1533945 (2019).

45. Zhu, C., Ren, Y. Y., Wu, J. N. \& Zhou, Q. J. A comparison of prediction of adverse perinatal outcomes between Hadlock and INTERGROWTH-21(st) Standards at the Third Trimester. Biomed. Res. Int. 2019, 7698038. https://doi.org/10.1155/2019/76980 38 (2019).

46. Poljak, B., Agarwal, U., Jackson, R., Alfirevic, Z. \& Sharp, A. Diagnostic accuracy of individual antenatal tools for prediction of small-for-gestational age at birth. Ultrasound Obstet. Gynecol. 49, 493-499. https://doi.org/10.1002/uog.17211 (2017).

47. Nordic perinatal statistics 2018. (Finnish institute for health and welfare, http://urn.fi/URN:NBN:fi-fe2020050525095, 2020). (Accepted on 27 May 2021).

48. Gabbay-Benziv, R. et al. Pregnancy outcome after false diagnosis of fetal growth restriction. J. Matern.-Fetal Neonatal Med. 30, 1916-1919. https://doi.org/10.1080/14767058.2016.1232383 (2017).

\section{Acknowledgements}

We are very grateful to all the women who participated in the study. We would also like to thank the Head of Department at the Women's Clinic in the five study sites for lending us your ultrasound machines and thereby making this project possible. We acknowledge the participating study sonographers: Pernilla Juthe (Uppsala), Anita Winter (Uppsala), Anne-Marie Drake (Falun), Christina von Feilitzen (Katrineholm), Bodil Edvardsson (Västerås), Annelie Trimmel (Örebro) and Frida Södergren (Örebro). The study was funded by the UppsalaÖrebro Regional Research Council, Region Uppsala Research and Development Grant, Födelsefonden, the Gillbergska Foundation and the Swedish Society of Medicine.

\section{Author contributions}

Study design and methodology: L.L., M.A., O.A., E.B. Project administration: L.L., E.B. Data management: L.L., M.A. Statistical analyses: L.L., M.A., L.H. Writing of original draft: L.L., M.A., O.A., L.H., A.S., A.K.W., E.B.

\section{Funding}

Open access funding provided by Uppsala University.

\section{Competing interests}

The authors declare no competing interests.

\section{Additional information}

Supplementary Information The online version contains supplementary material available at https://doi.org/ 10.1038/s41598-021-92032-2.

Correspondence and requests for materials should be addressed to L.L.

Reprints and permissions information is available at www.nature.com/reprints.

Publisher's note Springer Nature remains neutral with regard to jurisdictional claims in published maps and institutional affiliations. 
(c) (i) Open Access This article is licensed under a Creative Commons Attribution 4.0 International cc) License, which permits use, sharing, adaptation, distribution and reproduction in any medium or format, as long as you give appropriate credit to the original author(s) and the source, provide a link to the Creative Commons licence, and indicate if changes were made. The images or other third party material in this article are included in the article's Creative Commons licence, unless indicated otherwise in a credit line to the material. If material is not included in the article's Creative Commons licence and your intended use is not permitted by statutory regulation or exceeds the permitted use, you will need to obtain permission directly from the copyright holder. To view a copy of this licence, visit http://creativecommons.org/licenses/by/4.0/.

(C) The Author(s) 2021 